\title{
CS Research Square \\ Healthy Protection Of Bergamot Is Linked To The Modulation Of Microbiota
}

Antonella Riva ( $\square$ antonella.riva@indena.com )

Indena SpA https://orcid.org/0000-0003-2819-943X

Valeria Longo

Indena SpA

Davide Berlanda

Indena SpA

Pietro Allegrini

Indena SpA

Giulia Masetti

Parco tecnologico padano

Sara Botti

Parco tecnologico padano

Giovanna Petrangolini

Indena SpA

Research article

Keywords: microbiota, Bergamot Phytosome, Vazguard, metagenomics sequence, cardiovascular health

Posted Date: May 11th, 2020

DOI: https://doi.org/10.21203/rs.3.rs-25708/v1

License: (c) (i) This work is licensed under a Creative Commons Attribution 4.0 International License.

Read Full License 


\section{Abstract}

Background: The present study aimed to evaluate the effects of a new food-grade bioavailable delivery system of bergamot on human gut microbiota, in order to demonstrate the potential correlation of microbiota modulation in cardiovascular health.

The identification of human gut microbiota modification was performed after ex-vivo incubation with bergamot phytosome (Vazguard ${ }^{\mathrm{TM}}$ ) of individual faecal slurries from healthy women (45-53 years) as follows: after incubation at $37^{\circ} \mathrm{C}$ in anaerobic condition, DNA was extracted and a $16 \mathrm{~S}$ Metagenomic Sequencing Analysis performed.

Results: Twenty-five different phyla were identified, among which 4 were modulated: Firmicutes, Proteobacteria, Bacteroidetes, Actinobacteria. The decreased Firmicutes/Bacteroidetes ratio and the increase of Proteobacteria were observed indicating a positive modulation of microbiota possibly linked to cardiovascular health. 418 different genera were also identified, among which several of them were mildly modulated.

Conclusions: For the first time, a gut microbiome modulation was associated to the new delivery system of bergamot phytosome, supporting its clinical efficacy for cardiovascular health. New potential applications in weight control and gastrointestinal benefits were suggested.

\section{Background}

Intestinal microbiota is a complex ecosystem of microorganisms (such as bacteria, archeaebacteria, fungi and protists) resident in the gut [1]; their density and composition increase from stomach to colon and in the colon, about $10^{11}$ to $10^{12} / \mathrm{ml}$ are the estimated bacteria content [2,3]. A great number of studies have been dedicated in the last 15 years to explore human microbiota and its relationship with human diseases, such as cardiovascular and neurological disorders, inflammation, cancer, diabetes, and obesity [4], in order to identify modifications in gut bacterial composition possibly involved in a disease onset.

The high level of a mixture of flavonoids contained in the juice, albedo and flavedo of bergamot fruit is responsible for bergamot antioxidant and antisenescence properties [5]. Other beneficial effects such as hypoglycaemic and hypolipidaemic have been described for bergamot in metabolic syndrome and in cardiovascular diseases (CVD) prevention [6, 7].

A lecithin food-grade delivery system (Phytosome ${ }^{\circledR}$ ) containing the Bergamot Polyphenolic Fraction (BPF) from Citrus Bergamia Risso et Poiteau from Calabria was developed by Indena SpA to ameliorate the oral absorption of flavonoids. The structural phytochemical composition of bergamot was deeply analysed by HPLC-DAD-MS and LC-NMR [8], and a recent clinical trial in individuals with type 2 diabetes mellitus and hyperlipaemia showed a reduction of cardiovascular risk by modulating total cholesterol 
(tChol), low-density Lipoproteins (LDL), triglycerides (TG), high-density Lipoproteins (HDL) and blood glucose after just 30-day supplementation [9].

The aim of the present work is to study for the first time the effect of bergamot phytosome on human gut microbiota to find possible associations with its benefits on cardiovascular health.

\section{Results}

The $16 \mathrm{~S}$ Metagenomic results were analysed in order to highlight the potential gut microbiota modulation produced by incubation of faecal samples with the bergamot phytosome.

Shannon Index as a measure of the entropy of Species-level classifications [10] for both test and control groups (Fig. 1) was comprised between 1.5 and 3.5, indicating a good evenness on samples.

By using Illumina 16S Metagenomics workflow analysis, 25 different phyla were identified, with Firmicutes, Proteobacteria, Bacteroidetes and Actinobacteria as the four major phyla, as described in Fig. 2. The bergamot phytosome induced a decrease in the Firmicutes/Bacteroidetes ratio from 2.27 (Control) to 1.79 (test). Considering that an increase of the F/B ratio is correlated with obesity [3, 11], bergamot phytosome is endowed with a great potential for counteracting body fat accumulation.

A significant increase of Proteobacteria in bergamot phytosome treated samples was also observed ( $\mathrm{p}<$ 0.01 vs control), as reported in Fig. 3.

The Actinobacteria is the less represented phylum. Also in this case, an increased level was observed after bergamot phytosome incubation.

The analysis allowed identification of 418 different genera. Among them, 8 major genera (representing the $62 \%$ of those detected) were modulated, i.e. Escherichia, Serratia, Bacteroides, Prevotella, Enterococcus, Bifidobacterium, Blautia and Faecalibacterium (Fig. 4). More in detail (Fig. 5), an increase was observed in Blautia, Bifidobacterium, Escherichia, Serratia and Bacteroides while a reduction was observed for Enterococcus, Prevotella, and Faecalibacterium. Among them the observed difference for Escherichia (bergamot-treated 23.65 \pm 2.32 ; control $17.34 \pm 2.00$ ) and Serratia (bergamot-treated $3.98 \pm$ 0.38 ; control $2.67 \pm 0.24)$ was statistically significant $(p<0.05)$.

\section{Discussion}

For the first time, the effects of a new food-grade bioavailable delivery system of bergamot formulated in phytosome on human gut microbiota were investigated and then correlated to cardiovascular health, obesity and gastrointestinal disorders, thus supporting bergamot phytosome benefits in these areas, due to its potential to modulate related human gut microbiota after incubation with individual faecal slurries from healthy volunteers. 
Cardiovascular diseases (CVDs) are the first cause of death at global level: the World Health Organization declared that about 17.9 million people died from CVDs in 2016, corresponding to $31 \%$ of all global deaths (https://www.who.int/news-room/fact-sheets/detail/cardiovascular-diseases-(cvds).

Several factors, like smoking and alcohol use, poor diet, and obesity represent the major risks for CVDs [12], so prevention measures are of high importance in limiting the onset of diseases.

A large number of studies over ten years $[13,14]$ suggested a link between obesity and gut microbiota, i.e. the plethora of microorganisms living in the gastrointestinal tract [1].

In animal models, adiposity and weight increase can be affected by intestinal microbes. Mice of identical genotype exposed to a different environments and fed by a high-fat diet develop a distinct arrangement of gut microbiota, which influences the development of obesity and metabolic syndrome [15]. An alteration in the gut barrier induced by microbiota changes due to a high-fat diet in mice was also previously demonstrated $[16,17]$, and that condition led to metabolic diseases.

A human study conducted in obese and non-obese Danish people analysed genes from gut microbial populations identified individuals with low bacterial richness having more adiposity and dyslipidaemia in respect to high bacterial richness people [18], confirming a link between gut microbiota composition and obesity.

A recent paper reported a decrease in both triglycerides and LDL-C after 8-week treatments of a nutraceutical mixture containing BPF in dislypidemic overweight patients [19]. A significant reduction of serum low density lipoprotein cholesterol (LDL-C) and triglyceride was also demonstrated by a new bergamot phytosome formulation [9]: in that clinical study, the delivery by phytosome allowed increased oral absorption of the naringin, the major component of bergamot, in respect to standard unformulated bergamot. Actually, phytosome techniques have recently demonstrated the successful delivering of other low-soluble natural compounds, like quercetin [20] and Coenzyme Q10 [21].

In this experimental study, we explored the hypothesis that bergamot phytosome would be able to modulate the human gut microbiome. Among the 25 phyla detected, a decrease in the Firmicutes/Bacteroidetes ratio was observed, indicating involvement of bergamot phytosome in the field of weight control. In fact, it is well known that the Firmicutes/Bacteroidetes ratio value increases in the presence of an obesity status [2,3].

The significant increase of Proteobacteria observed in bergamot phytosome-treated samples $(p<0.01 v s$ control) supports a positive effect for health status: a decrease in Proteobacteria was associated with an increased risk of cardiovascular diseases [22].

When analysis of genus was performed, an increased modulation in Blautia was observed in bergamottreated samples. Blautia, involved in complex carbohydrate digestion, is recognized as a good indicator of the intestinal health, and its increase is beneficial. On the contrary, a Blautia reduction was observed in patients affected by heart failure [23], by hepatic and Chron's disease, and cancer [24-26]. Very 
interestingly, it has just been published that Blautia genus is inversely related with visceral fat accumulation [27].

Data obtained in the present study reported an increase in Bifidobacterium in bergamot-treated samples, suggesting again a positive modulation in weight control, because Bifidobacterium is decreased in obese subjects [28]. In our experiment, a reduction in Faecalibacterium was also observed: in the same paper of Gao the reduction is found in obese subjects, indicating for the latter a non-positive balance. However, in a very recent paper [29] an increase in Faecalibacterium prausnitzii is believed to be a good indicator of a health condition. In this regard, we must to underline that a large number of study on microbiome in different conditions (such as healthy or ill population, gender differences) do not allow the formulation of unique conclusions. In the same way Escherichia and Serratia increase (observed in bergamot-treated samples) would appear a non-positive effect.

The advantage in the present study of using fresh faecal materials from healthy subjects is undoubted, in respect to animal models. The study otherwise has some limitations due to its pilot design: the limited number of subjects, and the treatment administered via 'incubation' in laboratory samples. Further clinical studies are needed to better explore the benefits of bergamot phytosome related to microbiome modulation, even though encouraging preliminary indications in the present study suggested a potential positive modulation of microbiota for CVD by bergamot phytosome and give a strong rational for further exploration.

\section{Conclusions}

In conclusion, for the first time, a microbiome modulation was associated to bergamot phytosome, supporting preliminary evidence of its clinical benefit for cardiovascular health and opening interesting new applications.

\section{Methods}

\section{Samples collection}

Faecal samples from 3 healthy women aged $45-53$ years were collected in sterile containers, stored at $-20^{\circ} \mathrm{C}$ and then transferred at $-80^{\circ} \mathrm{C}$ until analysis. All subsequent methodologies were performed under anaerobic conditions to preserve the microbiota environmental. Donors had not used antibiotics in the previous 12 months.

\section{Phytosome digestion}

Bergamot Phytosome ${ }^{\circledR}($ Vazguard $\AA$, Indena SpA) was used in gut microbiota experiments. A simulated gastric and duodenal human digestion of bergamot phytosome $(1000 \mathrm{mg} / \mathrm{L})$ was performed in vitro before incubation with faecal slurries, mimicking in vivo conditions. Briefly, phytosome was subjected to simulated gastric digestion by incubation at $37^{\circ} \mathrm{C}$ for 60 minutes under shaking at $\mathrm{pH} 2$ with $\mathrm{HCl} 1 \mathrm{M}$ (to 
simulate gastric conditions before meal under a fasting state) in the presence of the gastric enzyme pepsin [30]. Further incubation under shaking for 150 minutes at $\mathrm{pH} 7.0$ with Sodium Carbonate $1 \mathrm{M}$ and in the presence of bile extract pancreatin (ratio 6:1) was performed to simulate pancreatic digestion [31]. The bile extract contains glycine, taurine and the main biliar salts, while pancreatin was utilized as source of pancreatic lipases and colipases.

\section{Ex-vivo incubation and samples analysis}

Faecal slurries $(1 \% \mathrm{w} / \mathrm{v})$ from each individual were used to inoculate the batch-culture system containing the nutrient medium and the digested bergamot phytosome (test). A batch culture system without bergamot phytosome and a non-treated faecal slurry sample were also included in the experiments as negative background control. After $16 \mathrm{~h}$ of incubation at $37^{\circ} \mathrm{C}$ under anaerobic condition, samples were centrifuged and DNA extracted. 16S Amplicon barcoded library were prepared and DNA sequence obtained by Next Generation Sequence utilizing the Miseq platform (Illumina Inc, CA, USA).

\section{Bioinformatics Analysis}

The 16S Metagenomics analysis were performed by using taxonomic classification of 16S rRNA targeted amplicon reads using an Illumina-curated version of the GreenGenes taxonomic database. The classification was performed by using the Illumina $16 \mathrm{~S}$ Metagenomics workflow, as described in Wang Q. et al [32].

\section{Statistical Analysis}

Data were analysed by Student's t test.

\section{Abbreviations}

HPLC-DAD-MS

High-Pressure Liquid Chromatograph-Diode Array Detector-Mass Spectrometry LC-NMR

Liquid Chromatography with Nuclear Magnetic Resonance Spectroscopy

tChol

total cholesterol

LDL

low-density Lipoproteins

TG

triglycerides

HDL

high-density Lipoproteins

CVD

Cardiovascular

F/B ratio 
Firmicutes/Bacteroidetes ratio

LDL-C

lipoprotein-cholesterol

\section{Declarations}

\section{Ethics Approval and Consent to participate}

The study was conducted in accordance with the Principles of Good Clinical Practice and the Declaration of Helsinki and was in agreement with the Ethical code of PTP Science Park (Lodi, Italy) (https://www.ptp.it/it/about-us\#governance). The collection of fecal samples from donors healthy volunteers was performed after Written informed consent in an anonimyzed way, and any information cannot be directly related to each volunteer [33].

\section{Consent for publication}

All authors of the above manuscript, as well as the responsible authorities at both institutes where the work has been carried out, have agreed to this submission, and agree to transfer the ownership of copyright to BMC Microbiology. If accepted, it will not be published elsewhere in the same form, in English or in any other language, including electronically without the written consent of the copyright-holder.

\section{Competing Interests}

AR, VL, DB, PA, GP are Indena's employees. GM and SB are employees at PTP Science Park.

\section{Funding}

This research did not receive any specific grant from funding agencies in the public, commercial or notfor-profit sectors.

\section{Authors' contributions}

Conceived and designed experiments: AR, GM, SB, DB, PA, GP. Performed the experiments: GM, SB. Analyze the data: VL, GP. Wrote the paper: AR, VL, DB, SB, GP. All the Authors read and approve the final manuscript.

\section{Availability of data and materials}

The datasets used and/or analysed during the current study are available from the corresponding author on reasonable request.

\section{Acknowledgements}

We would like to express our gratitude to Dr. Paola Misiano for her valuable editorial support. 


\section{References}

[1] E. Rinninella et al., "What is the Healthy Gut Microbiota Composition? A Changing Ecosystem across Age, Environment, Diet, and Diseases," (in eng), Microorganisms, vol. 7, no. 1, Jan 2019, doi: 10.3390/microorganisms7010014.

[2] R. E. Ley, F. Bäckhed, P. Turnbaugh, C. A. Lozupone, R. D. Knight, and J. I. Gordon, "Obesity alters gut microbial ecology," (in eng), Proc Natl Acad Sci U S A, vol. 102, no. 31, pp. 11070-5, Aug 2005, doi: 10.1073/pnas.0504978102.

[3] R. E. Ley, P. J. Turnbaugh, S. Klein, and J. I. Gordon, "Microbial ecology: human gut microbes associated with obesity," (in eng), Nature, vol. 444, no. 7122, pp. 1022-3, Dec 2006, doi: $10.1038 / 4441022 a$.

[4] P. D. Cani, "Human gut microbiome: hopes, threats and promises," (in eng), Gut, vol. 67, no. 9, pp. 1716-1725, 09 2018, doi: 10.1136/gutjnl-2018-316723.

[5] E. Da Pozzo et al., "Antioxidant and Antisenescence Effects of Bergamot Juice," (in eng), Oxid Med Cell Longev, vol. 2018, p. 9395804, 2018, doi: 10.1155/2018/9395804.

[6] M. Gliozzi et al., "Bergamot polyphenolic fraction enhances rosuvastatin-induced effect on LDLcholesterol, LOX-1 expression and protein kinase B phosphorylation in patients with hyperlipidemia," (in eng), Int J Cardiol, vol. 170, no. 2, pp. 140-5, Dec 2013, doi: 10.1016/j.ijcard.2013.08.125.

[7] P. P. Toth et al., "Bergamot Reduces Plasma Lipids, Atherogenic Small Dense LDL, and Subclinical Atherosclerosis in Subjects with Moderate Hypercholesterolemia: A 6 Months Prospective Study," (in eng), Front Pharmacol, vol. 6, p. 299, 2015, doi: 10.3389/fphar.2015.00299.

[8] C. Formisano et al., "Detailed Phytochemical Characterization of Bergamot Polyphenolic Fraction (BPF) by UPLC-DAD-MS and LC-NMR," (in eng), J Agric Food Chem, vol. 67, no. 11, pp. 3159-3167, Mar 2019, doi: 10.1021/acs.jafc.8b06591.

[9] V. Mollace et al., "Hypoglycemic and Hypolipemic Effects of a New Lecithin Formulation of Bergamot Polyphenolic Fraction: A Double Blind, Randomized, Placebo- Controlled Study," (in eng), Endocr Metab Immune Disord Drug Targets, vol. 19, no. 2, pp. 136-143, 2019, doi: 10.2174/1871530319666181203151513.

[10] M. O. Hill, "Diversity and Evenness: A Unifying Notation and Its Consequences," Ecology, vol. 54, no. 2, pp. 427-432, 1973, doi: 10.2307/1934352.

[11] A. Koliada et al., "Association between body mass index and Firmicutes/Bacteroidetes ratio in an adult Ukrainian population," (in eng), BMC Microbiol, vol. 17, no. 1, p. 120, 05 2017, doi: 10.1186/s12866017-1027-1. 
[12] S. E. Chiuve et al., "Lifestyle-based prediction model for the prevention of CVD: the Healthy Heart Score," (in eng), J Am Heart Assoc, vol. 3, no. 6, p. e000954, Nov 2014, doi: 10.1161/JAHA.114.000954.

[13] Y. Sanz, A. Santacruz, and G. De Palma, "Insights into the roles of gut microbes in obesity," (in eng), Interdiscip Perspect Infect Dis, vol. 2008, p. 829101, 2008, doi: 10.1155/2008/829101.

[14] P. Gérard, "Gut microbiota and obesity," (in eng), Cell Mol Life Sci, vol. 73, no. 1, pp. 147-62, Jan 2016, doi: 10.1007/s00018-015-2061-5.

[15] S. Ussar et al., "Interactions between Gut Microbiota, Host Genetics and Diet Modulate the Predisposition to Obesity and Metabolic Syndrome," (in eng), Cell Metab, vol. 22, no. 3, pp. 516-530, Sep 2015, doi: 10.1016/j.cmet.2015.07.007.

[16] M. Serino et al., "Metabolic adaptation to a high-fat diet is associated with a change in the gut microbiota," (in eng), Gut, vol. 61, no. 4, pp. 543-53, Apr 2012, doi: 10.1136/gutjnl-2011-301012.

[17] P. J. Turnbaugh, V. K. Ridaura, J. J. Faith, F. E. Rey, R. Knight, and J. I. Gordon, "The effect of diet on the human gut microbiome: a metagenomic analysis in humanized gnotobiotic mice," (in eng), Sci Transl Med, vol. 1, no. 6, p. 6ra14, Nov 2009, doi: 10.1126/scitranslmed.3000322.

[18] E. Le Chatelier et al., "Richness of human gut microbiome correlates with metabolic markers," (in eng), Nature, vol. 500, no. 7464, pp. 541-6, Aug 2013, doi: 10.1038/nature12506.

[19] A. F. G. Cicero, F. Fogacci, M. Bove, M. Giovannini, and C. Borghi, "Three-arm, placebo-controlled, randomized clinical trial evaluating the metabolic effect of a combined nutraceutical containing a bergamot standardized flavonoid extract in dyslipidemic overweight subjects," (in eng), Phytother Res, vol. 33, no. 8, pp. 2094-2101, Aug 2019, doi: 10.1002/ptr.6402.

[20] A. Riva, M. Ronchi, G. Petrangolini, S. Bosisio, and P. Allegrini, "Improved Oral Absorption of Quercetin from Quercetin Phytosome ${ }^{\circledR}$, a New Delivery System Based on Food Grade Lecithin," (in eng), Eur J Drug Metab Pharmacokinet, vol. 44, no. 2, pp. 169-177, Apr 2019, doi: 10.1007/s13318-018-0517-3.

[21] G. Petrangolini, M. Ronchi, E. Frattini, E. De Combarieu, P. Allegrini, and A. Riva, "A New Food-grade Coenzyme Q10 Formulation Improves Bioavailability: Single and Repeated Pharmacokinetic Studies in Healthy Volunteers," (in eng), Curr Drug Deliv, vol. 16, no. 8, pp. 759-767, 2019, doi:

$10.2174 / 1567201816666190902123147$.

[22] V. Dinakaran, A. Rathinavel, M. Pushpanathan, R. Sivakumar, P. Gunasekaran, and J. Rajendhran, "Elevated levels of circulating DNA in cardiovascular disease patients: metagenomic profiling of microbiome in the circulation," (in eng), PLoS One, vol. 9, no. 8, p. e105221, 2014, doi: 10.1371/journal.pone.0105221.

[23] M. Luedde et al., "Heart failure is associated with depletion of core intestinal microbiota," (in eng), ESC Heart Fail, vol. 4, no. 3, pp. 282-290, Aug 2017, doi: 10.1002/ehf2.12155. 
[24] J. Libertucci et al., "Inflammation-related differences in mucosa-associated microbiota and intestinal barrier function in colonic Crohn's disease," (in eng), Am J Physiol Gastrointest Liver Physiol, vol. 315, no. 3, pp. G420-G431, 09 2018, doi: 10.1152/ajpgi.00411.2017.

[25] T. H. Luu, C. Michel, J. M. Bard, F. Dravet, H. Nazih, and C. Bobin-Dubigeon, "Intestinal Proportion of Blautia sp. is Associated with Clinical Stage and Histoprognostic Grade in Patients with Early-Stage Breast Cancer," (in eng), Nutr Cancer, vol. 69, no. 2, pp. 267-275, 2017 Feb-Mar 2017, doi: 10.1080/01635581.2017.1263750.

[26] E. Nistal et al., "An altered fecal microbiota profile in patients with non-alcoholic fatty liver disease (NAFLD) associated with obesity," (in eng), Rev Esp Enferm Dig, vol. 111, no. 4, pp. 275-282, Apr 2019, doi: 10.17235/reed.2019.6068/2018.

[27] N. Ozato et al., "genus associated with visceral fat accumulation in adults 20-76 years of age," (in eng), NPJ Biofilms Microbiomes, vol. 5, p. 28, 2019, doi: 10.1038/s41522-019-0101-x.

[28] R. Gao et al., "Dysbiosis Signatures of Gut Microbiota Along the Sequence from Healthy, Young Patients to Those with Overweight and Obesity," (in eng), Obesity (Silver Spring), vol. 26, no. 2, pp. 351 361, 02 2018, doi: 10.1002/oby.22088.

[29] S. Verhoog et al., "Dietary Factors and Modulation of Bacteria Strains of," (in eng), Nutrients, vol. 11, no. 7, Jul 2019, doi: 10.3390/nu11071565.

[30] M. Minekus et al., "A standardised static in vitro digestion method suitable for food - an international consensus," (in eng), Food Funct, vol. 5, no. 6, pp. 1113-24, Jun 2014, doi: 10.1039/c3fo60702j.

[31] S. Hollebeeck, F. Borlon, Y. J. Schneider, Y. Larondelle, and H. Rogez, "Development of a standardised human in vitro digestion protocol based on macronutrient digestion using response surface methodology," (in eng), Food Chem, vol. 138, no. 2-3, pp. 1936-44, Jun 2013, doi:

10.1016/j.foodchem.2012.11.041.

[32] Q. Wang, G. M. Garrity, J. M. Tiedje, and J. R. Cole, "Naive Bayesian classifier for rapid assignment of rRNA sequences into the new bacterial taxonomy," (in eng), Appl Environ Microbiol, vol. 73, no. 16, pp. 5261-7, Aug 2007, doi: 10.1128/AEM.00062-07.

[33] A. Borovecki, A. Mlinaric, M. Horvat, V. Supak Smolcic. Informed consent and ethics committee approval in laboratory medicine. Biochem Med (Zagreb).2018 Oct 15;28(3):030201. doi:

10.11613/BM.2018.030201. Review.

\section{Figures}




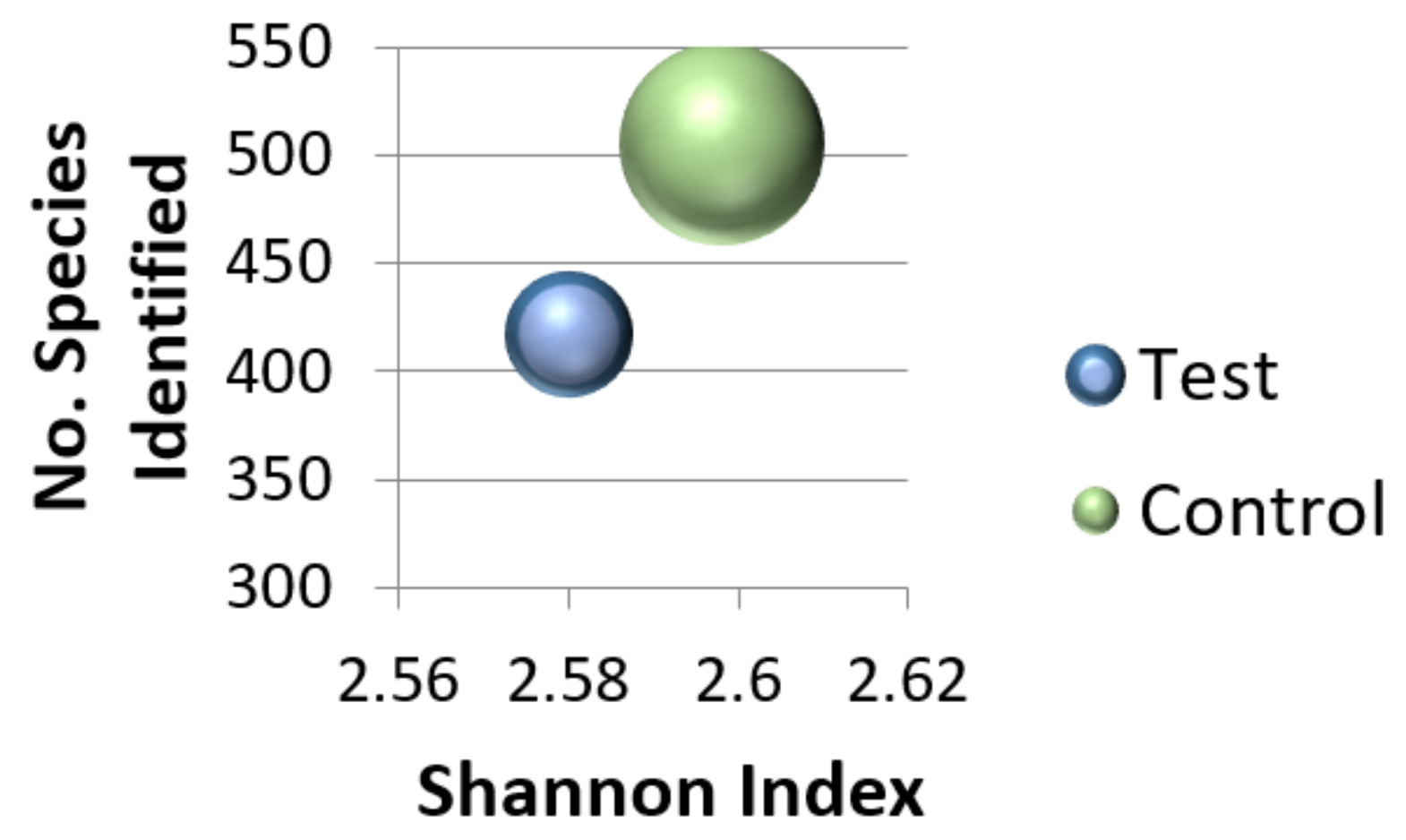

Figure 1

Shannon Index 


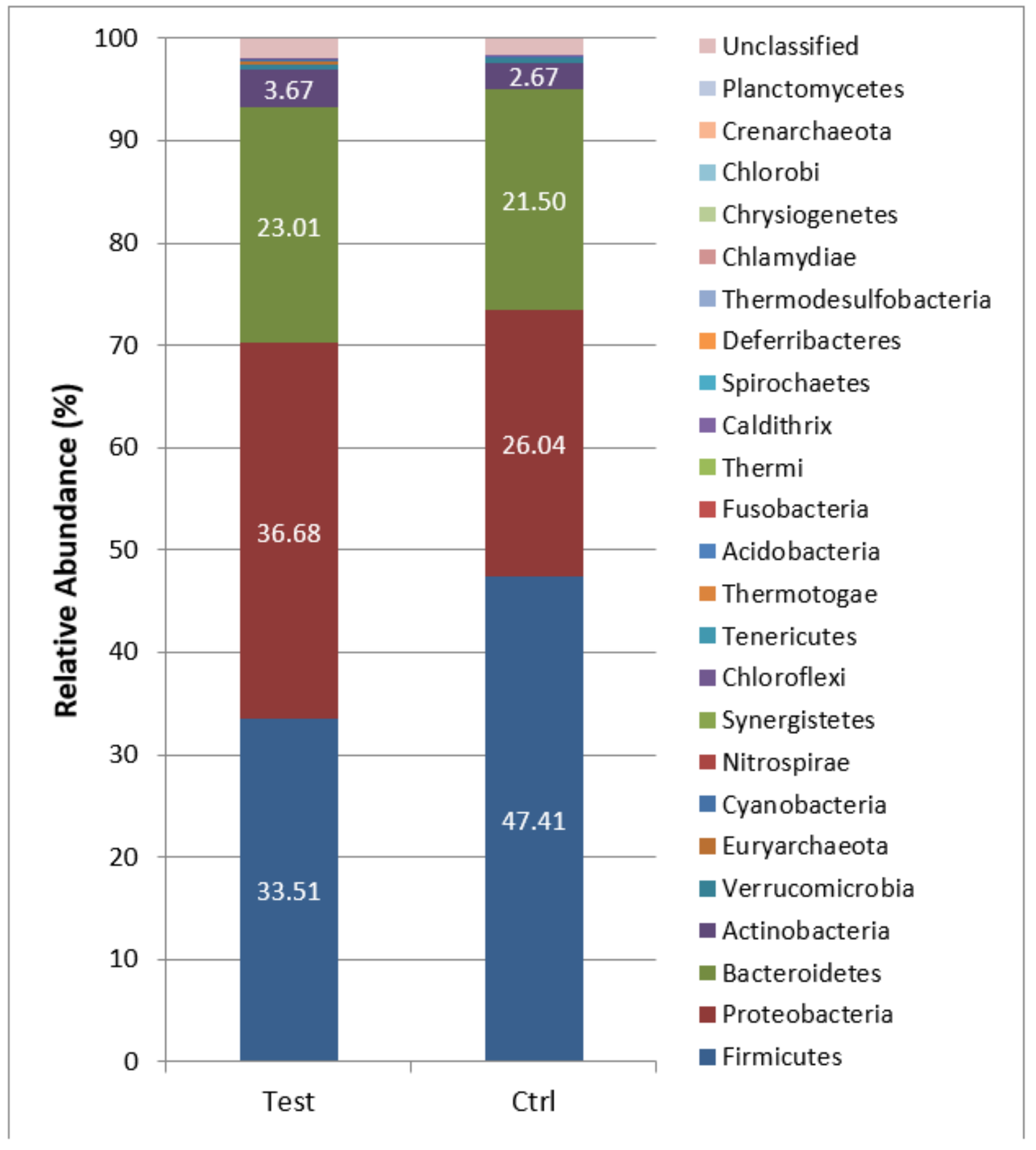

Figure 2

Relative Phyla abundance in control and treated faecal samples. 


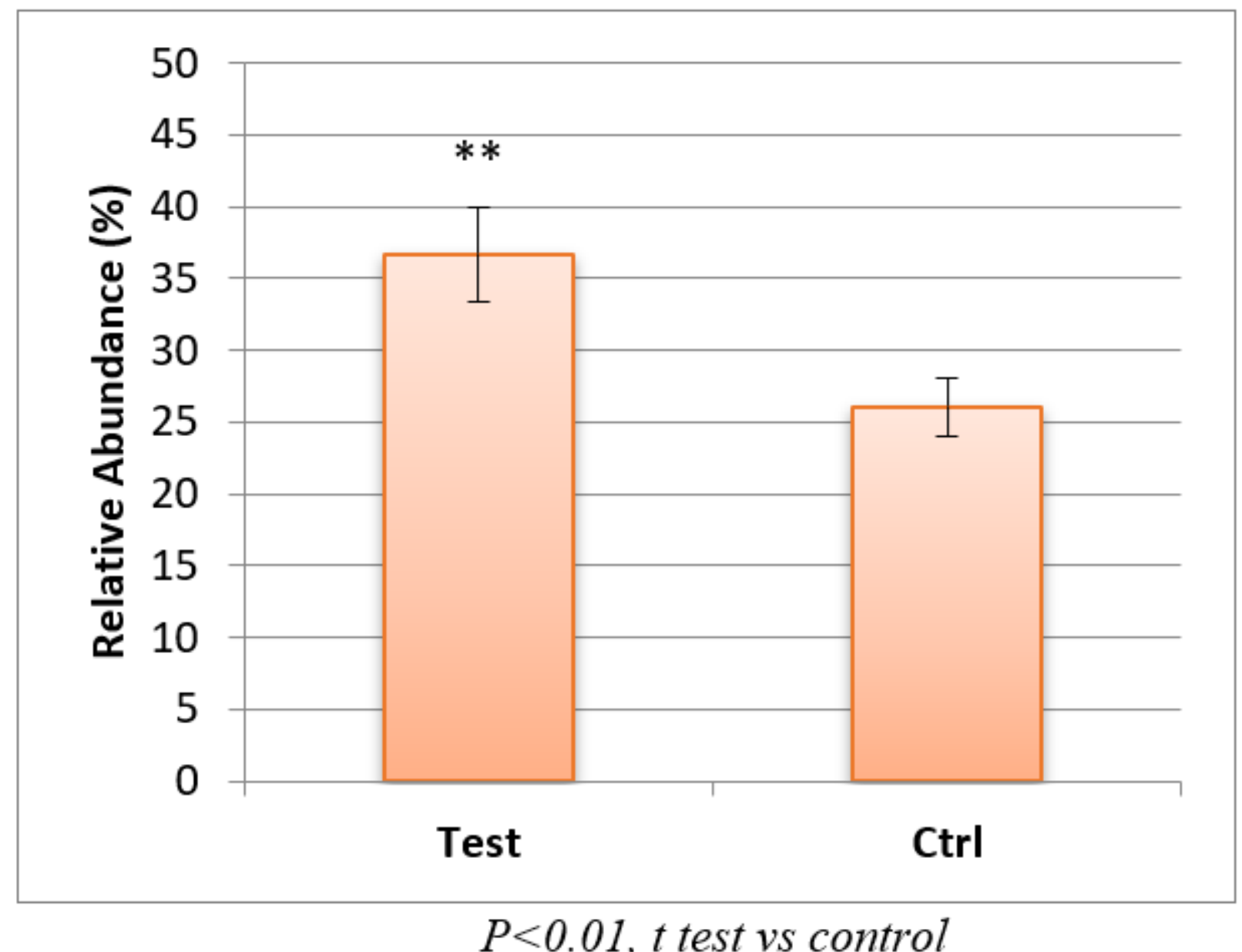

Figure 3

Proteobacteria levels in control and treated faecal samples. $\mathrm{P}<0.01, \mathrm{t}$ test vs control (Ctrl). 


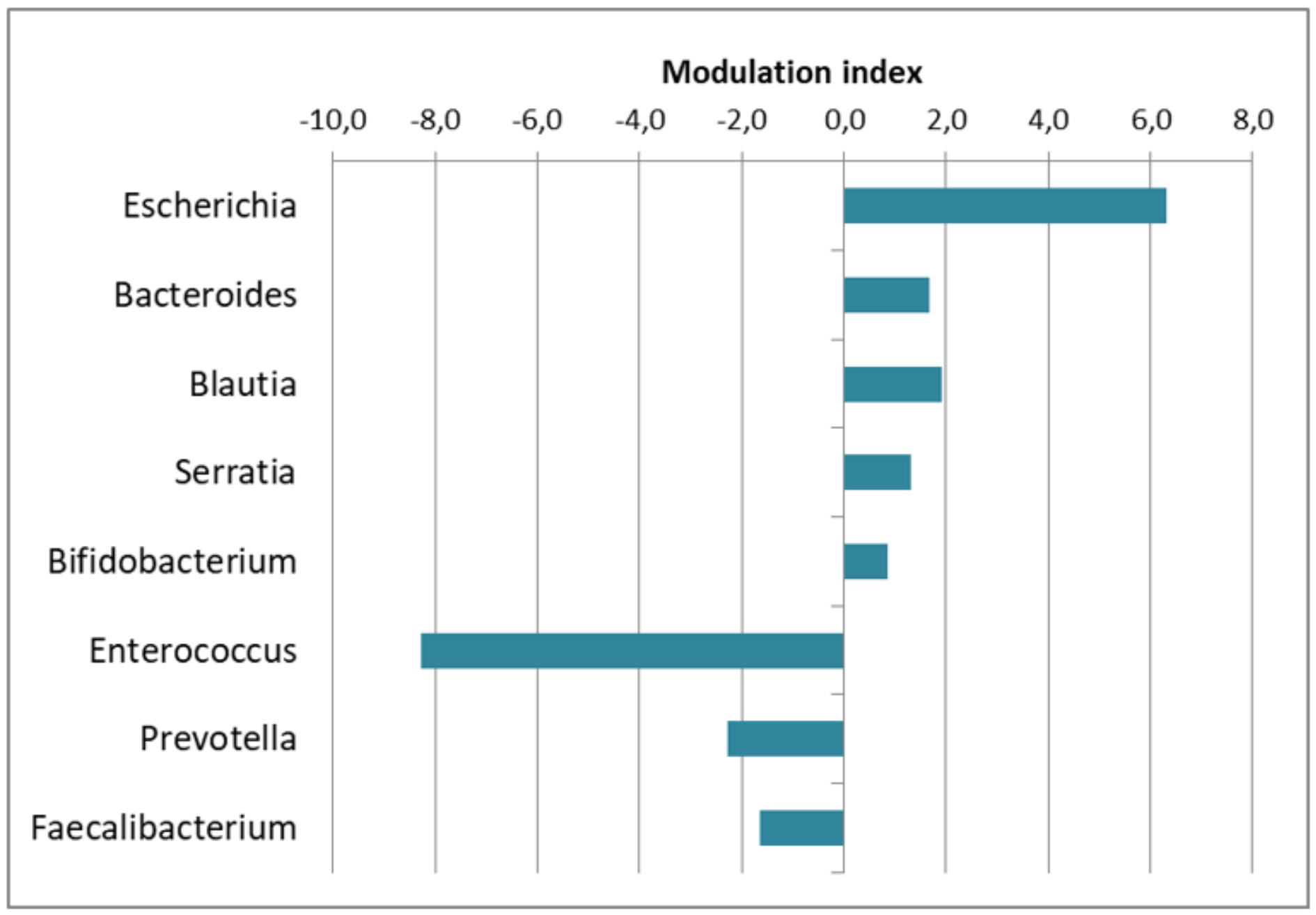

Figure 4

Summary of the Genera modulated by bergamot phytosome. 


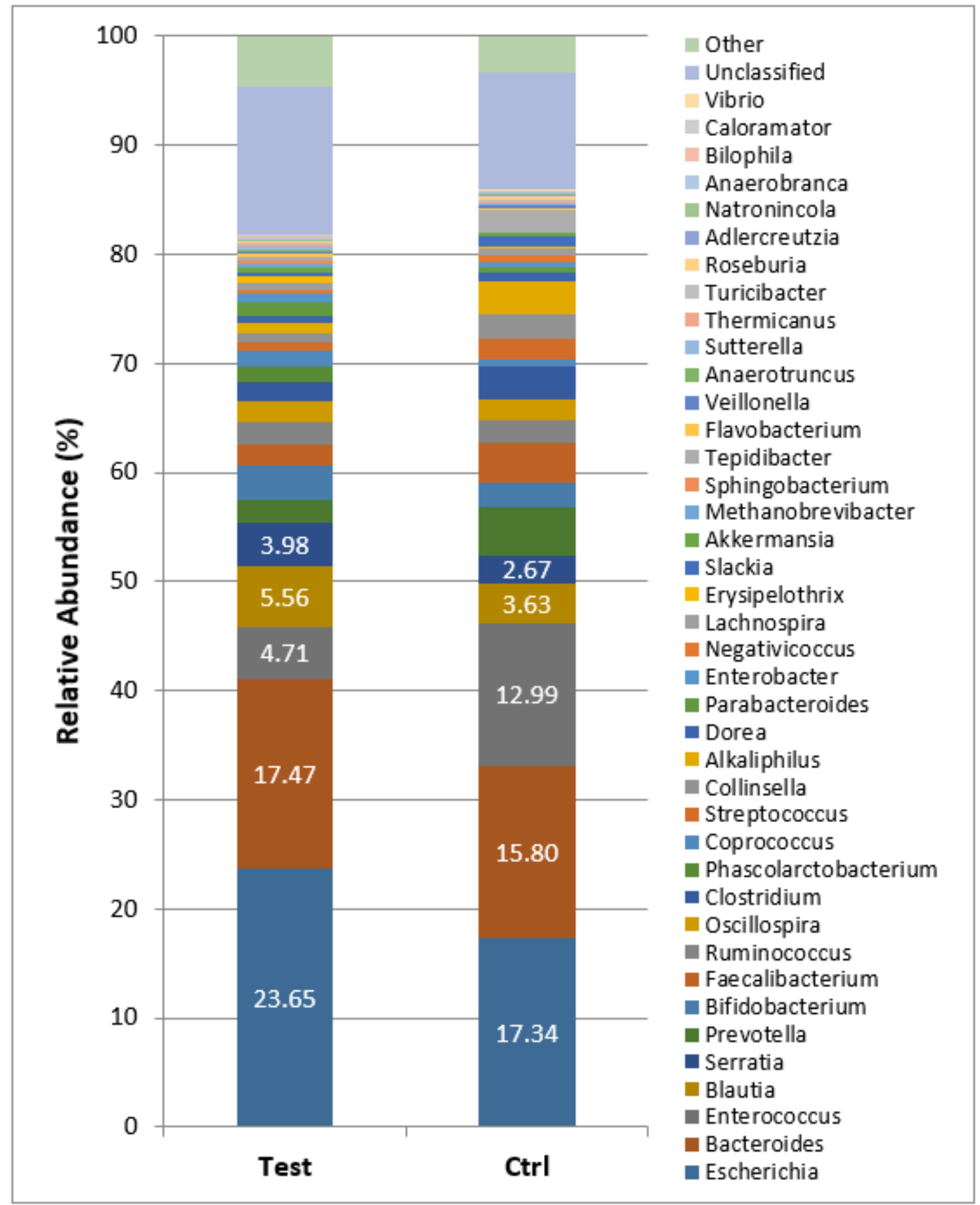

Figure 5

Relative Genera abundance in control and treated faecal samples. 